\title{
Antenatal corticosteroid administration and attention-deficit/hyperactivity disorder in childhood: a regression discontinuity study
}

\author{
Jennifer A. Hutcheon PhD, Erin C. Strumpf PhD, Jessica Liauw MD, M. Amanda Skoll MD, Peter Socha BSc, \\ Myriam Srour MD, Joseph Y. Ting MBBS, Sam Harper PhD
}

Cite as: CMAJ 2022 February 22;194:E235-41. doi: 10.1503/cmaj.211491

\begin{abstract}
Background: Antenatal corticosteroids reduce respiratory morbidity in preterm infants, but their use during late preterm gestation (34-36 weeks) is limited because their safety for longer-term child neurodevelopment is unclear. We sought to determine if fetuses with higher probability of exposure to antenatal corticosteroids had increased rates of prescriptions for attention-deficit/hyperactivity disorder (ADHD) medication in childhood, using a quasiexperimental design that better controls for confounding than existing observational studies.
\end{abstract}

Methods: We identified 16358 children whose birthing parents were admitted for delivery between $31+0$ (31 weeks, 0 days) and $36+6$ weeks' gestation in 2000-2013, using a perinatal data registry from British Columbia, Canada, and linked their records with population-based child ADHD medication data (2000-2018). We used a regression discontinuity design to capitalize on the fact that pregnancies presenting for delivery immediately before and immediately after the clinical cut-off for antenatal corticosteroid administration of $34+0$ weeks' gestation have very different levels of exposure to corticosteroids, but are otherwise similar with respect to confounders.

Results: Over a median follow-up period of 9 years, 892 (5.5\%) children had 1 or more dispensations of ADHD medication. Children whose birthing parents were admitted for delivery just before the corticosteroid clinical cut-off of $34+0$ weeks' gestation did not appear to be more likely to be prescribed ADHD medication than those admitted just after the cut-off (rate ratio $1.1,95 \%$ confidence interval $[\mathrm{Cl}] 0.8$ to $1.6 ; 1.3$ excess cases per 100 children, $95 \% \mathrm{Cl}-2.5$ to 5.7$)$.

Interpretation: We found little evidence that children with higher probability of exposure to antenatal corticosteroids have higher rates of ADHD prescriptions in childhood, supporting the safety of antenatal corticosteroids for this neurodevelopmental outcome.
A ntenatal corticosteroid administration to prevent neonatal respiratory morbidity and death has long been recommended for pregnancies with threatened preterm delivery before 34 weeks' gestation. ${ }^{1,2}$ Although evidence has shown that antenatal corticosteroids are also effective in preventing neonatal morbidity at late preterm gestation (34-36 weeks), ${ }^{3}$ their routine use at these ages is more controversial. ${ }^{4,5}$ Serious respiratory morbidity and death become less common with advancing gestation, ${ }^{6,7}$ so the potential harms of corticosteroids, including their unknown impact on child neurodevelopment, become a more important consideration than earlier in gestation..$^{8-10}$

High-quality evidence on the safety of antenatal corticosteroid administration for child neurodevelopment is scarce. Evidence from follow-up of randomized trials has generally been reassuring, but is limited by high losses to follow-up and small sample sizes. ${ }^{11-14}$ Several observational studies have explored the association between antenatal corticosteroids and child neurodevelopment, ${ }^{15,16}$ but their findings are prone to confounding. ${ }^{5}$ Further, they have tended to focus on rare neurodevelopmental conditions such as cerebral palsy, ${ }^{15}$ and our understanding of the association with common neurodevelopmental outcomes such as attention-deficit/hyperactivity disorder (ADHD) is limited. Repeat courses of antenatal corticosteroids have been linked with increased hyperactivity in animal research ${ }^{17}$ and with increased attention problems in children at age 2 years in 1 randomized trial, ${ }^{18}$ supporting the importance of investigating this outcome.

In this study, we aimed to investigate whether children with a higher probability of in-utero exposure to antenatal corticosteroids were more likely to be prescribed ADHD medications. 


\section{Methods}

\section{Study design}

We used a regression discontinuity design, which is a quasiexperimental approach that exploits how treatment decisions often differ above and below a clinical cut-off or threshold that is somewhat arbitrary. Individuals immediately on either side of the cut-off tend to be highly similar except for their probability of receiving treatment; therefore, comparisons of individuals immediately above and below the threshold can provide evidence for causal effects of the intervention that may be less confounded than direct comparisons of individuals who did or did not receive treatment, as in standard observational studies. ${ }^{19,20}$ For example, during our study period, a Canadian clinical practice guideline recommended that pregnancies with threatened preterm delivery should be treated with antenatal corticosteroids up to $33+6$ (33 weeks, 6 days) weeks' gestation, but not at $34+0$ weeks or later. ${ }^{21}$ Thus, infants just below and just above the 34-week threshold have very different probabilities of exposure to antenatal corticosteroids, but are otherwise very similar with respect to confounder characteristics.

Using the gestational age cut-off of $33+6$ weeks, we have previously shown that the regression discontinuity design can produce estimates of the short-term benefits of antenatal corticosteroids comparable to those of randomized trials, with a $19 \%$ lower risk of neonatal respiratory morbidity or death ${ }^{22}$ compared with the $20 \%$ reduction in the primary respiratory outcome reported in the Antenatal Late Preterm Steroids trial. ${ }^{3}$

\section{Study population}

We included all live, singleton births to pregnant individuals admitted for delivery between $31+0$ weeks' and $36+6$ weeks' gestation in British Columbia, Canada, from April 2000 to March 2013 (irrespective of reason for admission). We linked population-based medical records from the BC Perinatal Data Registry, which contains abstracted records for more than $99 \%$ of births in the province, with the PharmaNet register, which contains records of all outpatient prescriptions. ${ }^{23,24}$ We also linked coverage records and basic census geodata from BC Vital Statistics and Medical Services. ${ }^{25,26}$ Linkages were conducted using names, birthdates and Medical Services Plan numbers (a unique identifier for provision of universal health care). We obtained records from PharmaNet, BC Vital Statistics and Medical Services from April 2000 to March 2018, ensuring 5 or more years of follow-up for all individuals.

\section{Exposure}

Our primary exposure was gestational age at the time of hospital admission, as we hypothesized that this better approximated the timing of decision-making on antenatal corticosteroid administration than gestational age at delivery. Gestational age in days was estimated using last menstrual period, confirmed or revised with early ultrasonography. ${ }^{27}$

We limited our cohort to births with a day-specific, ultrasonography-confirmed estimate of gestational age. Validation work has established that about $85 \%$ of births at this time had a prenatal ultrasonography:28 however, day-specific estimates were available for only $56 \%$ of the population because, before 2008 , information on gestational age by ultrasonography was abstracted in completed weeks only, not days. Moreover, scans done after $20+0$ weeks are not abstracted. As most births with missing day-specific estimates from ultrasonography were missing owing to administrative reasons, we do not believe that this introduced meaningful bias. ${ }^{22}$

\section{Antenatal corticosteroid administration}

Antenatal corticosteroid administration is documented in the $\mathrm{BC}$ Perinatal Data Registry as a binary variable indicating administration of any corticosteroid during the delivery admission for the purpose of fetal lung maturation. We used this variable for descriptive purposes only, as our primary exposure was the gestational age at admission for delivery rather than individual-level corticosteroid exposure.

\section{Outcomes}

Our primary outcome was the dispensation of 1 or more medications categorized under N06B in the Anatomical Therapeutic Chemical classification, which corresponds to "psychostimulants, agents used for ADHD, and other nootropics." This category includes all first-line ADHD medications recommended by the Canadian Paediatric Society. ${ }^{29}$ We also evaluated 2 or more dispensations of N06B medications as a secondary outcome. We classified infant deaths as having experienced the outcome to avoid selection bias (i.e., infants who did not receive corticosteroids may not have survived to develop ADHD).

\section{Statistical analysis}

We calculated descriptive characteristics of the study population using means with standard deviations, medians with interquartile ranges (IQR) or frequencies with percentages. We calculated weekand day-specific proportions to describe patterns of antenatal corticosteroid administration and ADHD dispensations by gestational age. We used a regression discontinuity design ${ }^{30}$ to compare rates of ADHD prescriptions among children whose birthing parents were admitted just before and just after $34+0$ weeks' gestation $(<34+0$ v. $\geq 34+0$ weeks). The model effectively estimates the effect of routine antenatal corticosteroid administration as the difference in outcome rate immediately before versus immediately after the discontinuity, controlling for underlying gestational age trends before and after the discontinuity separately (illustrated in Appendix 1, Figure S1, available at www.cmaj.ca/lookup/ doi/10.1503/cmaj.211491/tab-related-content). The estimate produced by the model is analogous to an intention-to-treat effect, because it reflects the impact of antenatal corticosteroid administration as it occurs in a real-world setting (in which imperfect adherence to corticosteroid recommendations occurs because of insufficient time to administer corticosteroids before delivery, incomplete courses and mistimed administration).

We implemented our regression discontinuity model using pooled log binomial regression. Individuals were censored the end of follow-up (Mar. 31, 2018) or if they moved out of province. We used triangular weighting to assign the greatest weight to infants delivered immediately on either side of the cut-off at $34+0$ weeks, and the least weight to those furthest away from the cut-off ( $31+0$ and $36+6$ weeks). We bootstrapped $95 \%$ confidence intervals (Cls). We used the model coefficients to estimate the number of excess 
ADHD prescriptions per 100 children by age 13 years (i.e., the absolute difference in cumulative risk) at the point of the discontinuity, with bootstrapped $95 \% \mathrm{Cls}$. Our pre-analysis plan and statistical code are available online (https://osf.io/4exfq/).

\section{Sensitivity analyses}

We conducted standard checks to verify model assumptions. ${ }^{31}$ We evaluated trends in maternal-fetal characteristics to ensure that they changed smoothly across gestation, evaluated whether a quadratic term for gestational age improved model fit, and altered the gestational age time window (bandwidth) on either side of $34+0$ weeks included in our analyses. Finally, we controlled for male fetal sex as a confounder and included a product term between sex and before and after the cut-off.

\section{Ethics approval}

The study was approved by the BC Children's Hospital Research Ethics Board (\#H18-00620).

\section{Results}

We identified 294807 live, singleton births with a day-specific, ultrasonography-confirmed estimate of gestational age. We excluded 278449 births (94.5\%) admitted for delivery after $36+6$ weeks' or before $31+0$ weeks' gestation, leaving 16358 (5.5\%) pregnancies for analysis. Over the 16358 pregnancies, $2594(15.8 \%)$ were admitted before $34+0$ weeks and 13764 $(84.1 \%)$ were admitted at $34+0$ weeks or later. The median duration of follow-up was 9 (IQR 7-13) years.

Table 1: Descriptive characteristics of pregnancies admitted for delivery between $31+0$ and $36+6$ weeks' gestation, British Columbia, Canada, 2000-2013

No. (\%) of pregnancies*

\begin{tabular}{|c|c|c|c|}
\hline Characteristic & $\begin{array}{c}\text { Total } \\
n=16358\end{array}$ & $\begin{array}{l}\text { Admission before } 34 \text { wk } \\
\qquad n=2594\end{array}$ & $\begin{array}{l}\text { Admission at or after } 34 \mathrm{wk} \\
\qquad n=13764\end{array}$ \\
\hline Age of gestational parent, $\mathrm{yr}$, mean \pm SD & $31.2 \pm 5.7$ & $31.5 \pm 5.7$ & $31.1 \pm 5.7$ \\
\hline Nulliparous & $8129(49.7)$ & $1367(52.7)$ & $6762(49.1)$ \\
\hline Pre-pregnancy BMI $\left(\mathrm{kg} / \mathrm{m}^{2}\right) \dagger$ & $24.5 \pm 5.5$ & $24.5 \pm 5.4$ & $24.4 \pm 5.5$ \\
\hline Smoking in pregnancy & $1878(11.5)$ & $288(11.1)$ & $1590(11.6)$ \\
\hline \multicolumn{4}{|l|}{ Neighbourhood income quintile } \\
\hline 1 (lowest) & $3581(21.9)$ & $551(21.7)$ & $3030(22.0)$ \\
\hline 2 & 3419 (20.9) & $587(23.1)$ & $2832(20.6)$ \\
\hline 3 & $3235(19.8)$ & $493(19.4)$ & $2742(19.9)$ \\
\hline 4 & $3115(19.0)$ & $466(18.3)$ & $2649(19.3)$ \\
\hline 5 (highest) & $2401(14.7)$ & $383(15.1)$ & $2018(14.7)$ \\
\hline Missing & $607(3.7)$ & $114(4.4)$ & $493(3.6)$ \\
\hline Hypertensive disorder of pregnancy & $2543(15.6)$ & $501(19.3)$ & $2042(14.8)$ \\
\hline Diabetes in pregnancy & $2811(17.2)$ & $499(19.2)$ & $2312(16.8)$ \\
\hline latrogenic delivery $\ddagger$ & $7204(44.0)$ & $1151(44.4)$ & $6053(44.0)$ \\
\hline \multicolumn{4}{|l|}{ Gestational age at delivery, wk } \\
\hline 31 & $350(2.1)$ & $350(13.5)$ & - \\
\hline 32 & $629(3.9)$ & $629(24.3)$ & - \\
\hline 33 & $1074(6.6)$ & $1074(41.4)$ & - \\
\hline 34 & $1942(11.9)$ & $357(13.8)$ & $1585(11.5)$ \\
\hline 35 & $3497(21.4)$ & $93(3.6)$ & $3404(24.7)$ \\
\hline 36 & $7806(47.7)$ & $51(2.0)$ & $7755(56.3)$ \\
\hline 37 & $936(5.7)$ & $24(0.9)$ & $912(6.6)$ \\
\hline 38 & $79(<1)$ & $8(0.3)$ & $71(0.5)$ \\
\hline$\geq 39$ & $45(<1)$ & $8(0.3)$ & $37(0.3)$ \\
\hline Male fetus & $9243(56.5)$ & $1522(58.7)$ & $7721(56.1)$ \\
\hline Birthweight, g, mean \pm SD & $2671 \pm 534$ & $2110 \pm 486$ & $2777 \pm 473$ \\
\hline \multicolumn{4}{|l|}{$\begin{array}{l}\text { Note: } \mathrm{BMI}=\text { body mass index, SD = standard deviation. } \\
\text { *Unless indicated otherwise. } \\
\text { †Among } 11973 \text { pregnancies with BMI available. } \\
\text { †Delivery after prelabour cesarean or labour induction. }\end{array}$} \\
\hline
\end{tabular}


As expected for a cohort of preterm births, hypertensive disorders of pregnancy and diabetes were common $(n=2543,15.6 \%$ and $n=2811,17.2 \%$, respectively), and 7204 (44.0\%) infants had an iatrogenic delivery (Table 1 ). Although pregnancies admitted before $34+0$ weeks were moderately more likely than those admitted 34 weeks or later to be nulliparous (53.0\% v. 49.3\%, respectively), have a hypertensive disorder of pregnancy $(19.3 \% \mathrm{v} .14 .8 \%$, respectively) or have diabetes ( $19.2 \%$ v. $16.8 \%$, respectively), these characteristics (and others) changed smoothly across our gestational age range (Appendix 1, Figure S2), with no abrupt changes at $34+0$ weeks' gestation that could introduce confounding.

\section{Patterns of antenatal corticosteroid administration}

Pregnancies admitted before $34+0$ weeks' gestation were 24 times more likely to be exposed to corticosteroids than those admitted later (Figure 1). Antenatal corticosteroid administration decreased sharply at $34+0$ weeks, from about $50 \%$ of pregnancies admitted before $34+0$ to less than $2 \%$ for those admitted after the cut-off. The decrease occurred primarily over a 4-day period for admissions between $33+4$ and $34+0$ weeks.

\section{Patterns of attention-deficit/hyperactivity disorder medication dispensing}

About 1 in 20 children were prescribed 1 or more medications for ADHD, with a median age at first prescription of 8 years (Table 2). Three-quarters ( $n=676,75.7 \%$ ) of dispensation were to boys. Children with ADHD medication prescriptions filled a median of 11 dispensations, of which nearly two-thirds (63.9\%) were for methylphenidate hydrochloride.

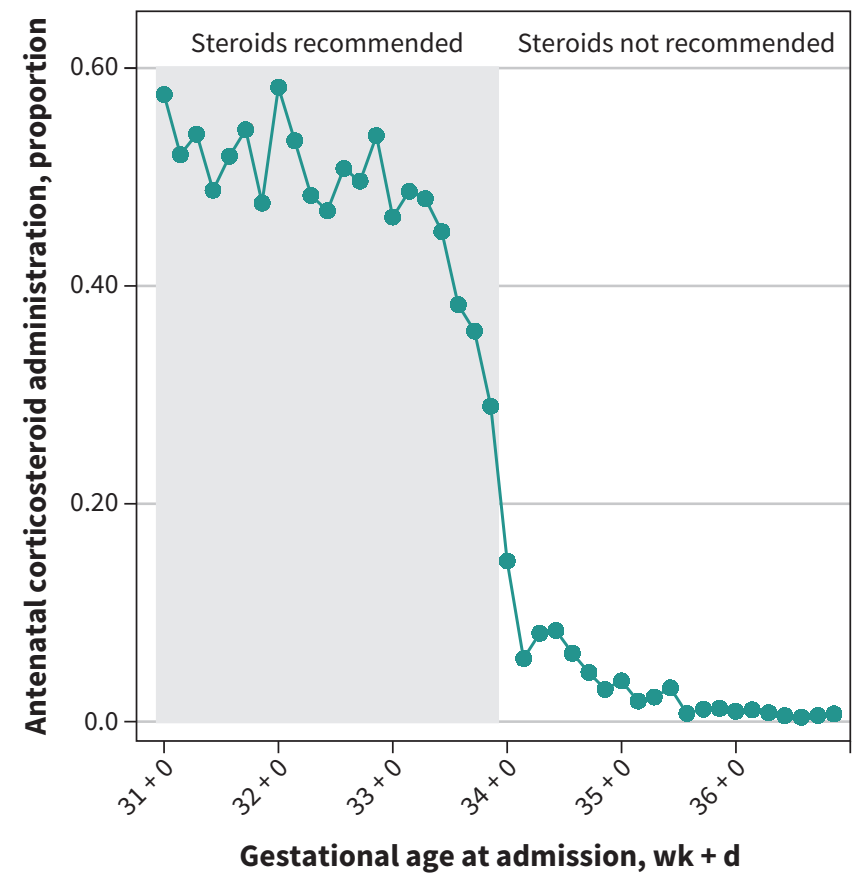

Figure 1: Rate of antenatal corticosteroid administration by gestational age at admission of 16358 pregnancies admitted for delivery between $31+0$ and $36+6$ weeks' gestation, British Columbia, Canada, 2000-2013.

\section{Regression discontinuity analysis}

Figure 2 shows the crude prevalence of ADHD prescriptions by gestational week at admission. Although prescriptions decreased steadily with advancing gestational age (from 9.4\% in children born at 31 weeks to $5.8 \%$ for those born at 36 weeks), no abrupt difference distinguished between children whose birthing parents were admitted for delivery just before versus just after $34+0$ weeks' gestation ( $6.8 \%$ at 33 weeks v. $6.8 \%$ at 34 weeks). This was confirmed in the regression discontinuity analysis (Figure 3 and Table 3), which found no clinically meaningful difference in ADHD medication dispensation rates between children whose birthing parents were admitted just before versus just after $34+0$ weeks (rate ratio $1.1,95 \% \mathrm{Cl} 0.8$ to $1.6 ; 1.3$ excess cases per 100 children by age $13,95 \% \mathrm{Cl}-2.5$ to 5.7 ). Results were similar for the secondary outcome of 2 or more dispensations.

\section{Sensitivity analyses}

Results were similar after shortening the gestational age window to 14 days on either side of the discontinuity of $34+0$ weeks (Appendix 1, Table S1). After shortening the gestational age window to 7 days, the model estimated a significantly increased risk of childhood prescriptions for ADHD medications among infants born just before $34+0$ weeks (rate ratio $2.5,95 \% \mathrm{Cl} 1.3$ to 4.4 ). However, as shown in Appendix 1, Figure S3, this result appeared to be driven by an estimated increase in risk between $33+0$ and $33+6$ weeks (i.e., an upward slope line), which is counter to the

Table 2: Patterns of childhood prescriptions of

medications for attention-deficit/hyperactivity disorder

among children of 16358 pregnancies admitted for

delivery between $31+0$ and $36+6$ weeks' gestation in

British Columbia, Canada, 2000-2013

\section{Characteristic}

$\geq 1$ prescription

Age at first prescription, yr, median (IQR) $\dagger$

$\geq 1$ prescription among children aged $\geq 13 \mathrm{yr} \ddagger$

No. of dispensations, median (IQR) $\dagger$

Estimated total number of $d$ of treatment (total $d$ supplied for all medications, per child), median (IQR)†

Type of medication, no. (\%) of total dispensations

Methylphenidate hydrochloride

Atomoxetine hydrochloride

Dextroamphetamine sulfate

Dextroamphetamine-amphetamine

Lisdexamfetamine dimesylate

Total dispensations

Note: IQR = interquartile range.

*Unless indicated otherwise.

†Among 892 children with at least 1 prescription.

$\ddagger$ Among 5458 children with 13 or more years of follow-up.
No. $(\%)$ of pregnancies ${ }^{\star}$ $n=16358$

$892(5.5)$

$8(6-10)$

$449(8.2)$

11 (4-31)

362 (118-1041) $12069(63.9)$ 1936 (10.3)

1717 (9.1) $939(5.0)$ $2223(11.8)$

18884 


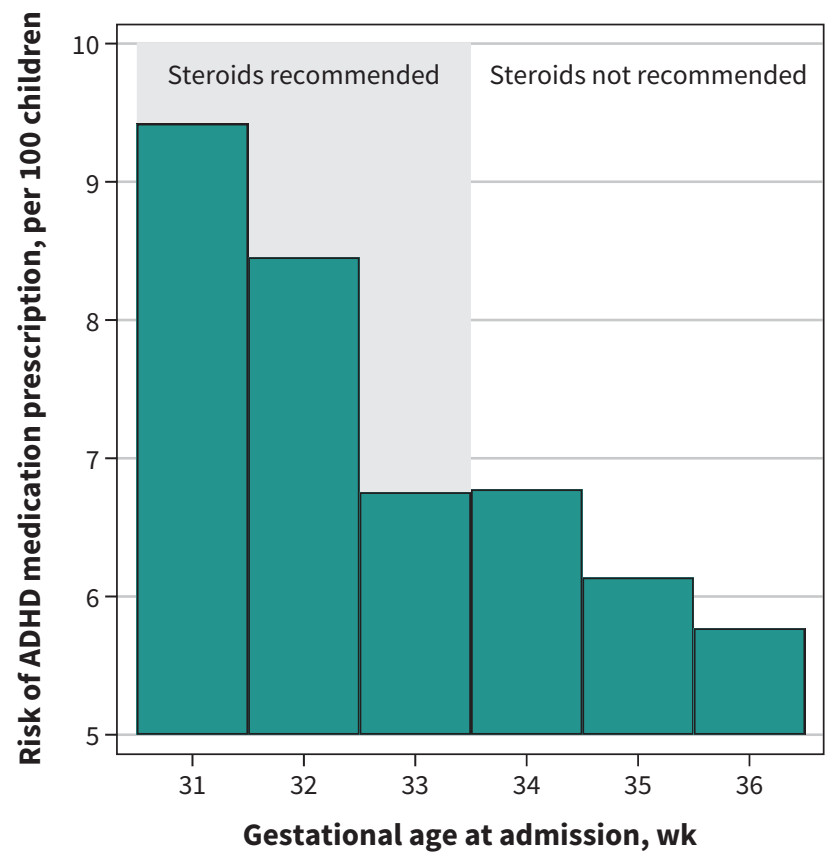

Figure 2: Risk of childhood prescription of medication for attentiondeficit/hyperactivity disorder (ADHD) per 100 children, by gestational age (in weeks) at hospital admission (between $31+0$ and $36+6$ weeks) for delivery of 16358 children in British Columbia, Canada, 2000-2013. more plausible pattern of decreasing risk in adverse neurodevelopmental outcomes with advancing gestational age. We therefore believe this result reflects numerical instability associated with estimating a trend using only 7 data points (days) rather than a true increase in risk. Controlling for sex in the model had no meaningful impact (Appendix 1, Table S1), nor was there evidence for effect measure modification by sex $(p=0.4)$.

\section{Interpretation}

In this population-based study of children whose birthing parents were admitted for delivery between $31+0$ and $36+6$ weeks' gestation, we found little evidence of an increased risk of ADHD medication prescription among children of pregnant individuals admitted for delivery just before the clinical cut-off for antenatal corticosteroid administration of $34+0$ weeks (i.e., with high probability of exposure to antenatal corticosteroids), compared with those admitted just after this cut-off (i.e., with minimal exposure to antenatal corticosteroids). These findings provide reassurance to clinicians and families concerned about the longer-term safety of antenatal corticosteroids for more subtle neurodevelopment outcomes such as ADHD.

Two cohort studies that followed patients who had completed randomized trials comparing single-course antenatal corticosteroids to placebo have evaluated subsequent risk of ADHD. A followup of the 1969-1974 Auckland steroid trial found no significant

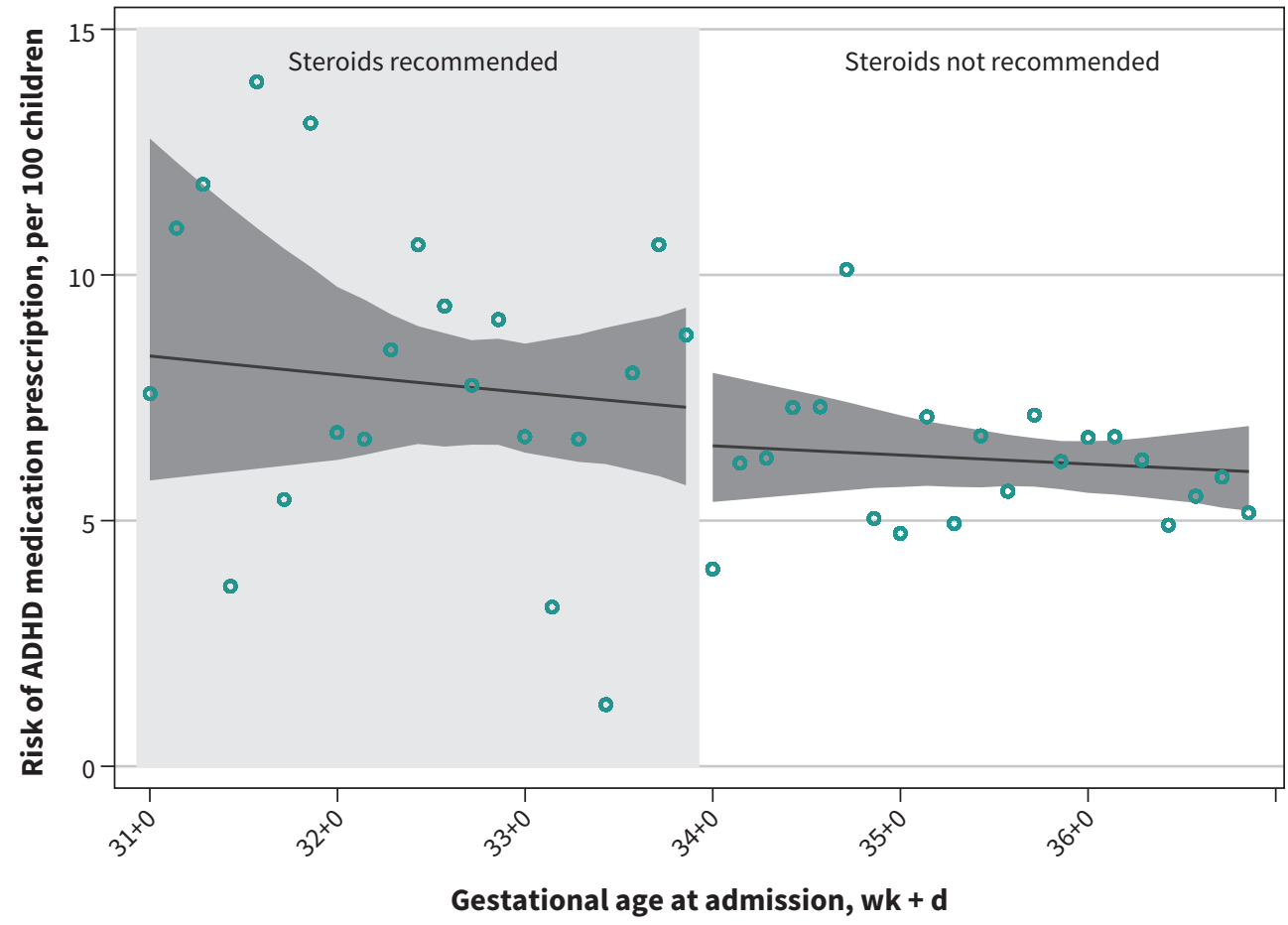

Figure 3: Risk of prescriptions of medication for attention-deficit/hyperactivity disorder (ADHD) per 100 children, by gestational age at hospital admission (between $31+0$ and $36+6$ weeks) for delivery of 16358 children in British Columbia, Canada, 2000-2013. Circles indicate observed day-specific values; solid line indicates the smoothed estimate of risk of prescription with $95 \%$ confidence interval (dark grey shaded area). 
Table 3: Estimated risk of childhood prescription of medications for attention-deficit/

hyperactivity disorder among children born to birth parents admitted for delivery between

$31+0$ and $33+6$ weeks' gestation versus those admitted between $34+0$ and $36+6$ weeks'

gestation in British Columbia, Canada, 2000-2013

\section{Prescription}

$\geq 1$ dispensation

$\geq 2$ dispensations
Rate ratio $(95 \% \mathrm{Cl})$

$1.12(0.8$ to 1.6$)$

$1.1(0.7$ to 1.7$)$
Excess cases per 100 children by 13 years of age* $(95 \% \mathrm{Cl})$

$1.3(-2.5$ to 5.7$)$

$1.3(-3.1$ to 5.6$)$

Note: $\mathrm{Cl}=$ confidence interval.

${ }^{*}$ Absolute difference in cumulative risk by age 13 .

difference in scores on the Brown attention-deficit disorder scale in individuals with and without prenatal exposure to betamethasone (mean scores 25.6 v. 26.9, respectively). ${ }^{12} \mathrm{~A}$ follow-up of the Antenatal Steroids for Term Elective Cesarean Section (ASTECS) trial also found no difference between study arms in hyperactivity subscale scores of a strengths and difficulties questionnaire. ${ }^{14}$ However, the evidence from these studies is limited by low followup rates ( $17 \%$ and $47 \%$, respectively) and small sample sizes ( $n=192$ and $n=407$, respectively). Our findings are consistent with these randomized studies in suggesting that antenatal corticosteroid exposure does not increase the risk of ADHD, but considerably strengthens the evidence behind this conclusion through our use of a large, population-based sample that reflects the real-world impact of antenatal corticosteroid administration, combined with a robust quasiexperimental design that reduces confounding. However, a recent population-based, observational study from Finland found that children exposed to antenatal corticosteroids had increased risks of receiving a diagnosis of ADHD or conduct disorder. ${ }^{16}$ Given that the effect size was small (a $20 \%$ increase in risk), confounding by indication could plausibly explain these findings. ${ }^{10}$

\section{Limitations}

Prescription of a medication used for ADHD is a different outcome than an ADHD diagnosis. A study from Ontario found that $70 \%$ of children with a chart-confirmed ADHD diagnosis were prescribed ADHD medications; ${ }^{32}$ thus, the outcome of ADHD medication prescriptions does not reflect the incidence of ADHD. However, unlike ADHD diagnosis, ADHD medication dispensation is accurately documented in population databases and is a clinically relevant outcome in its own right, making it a more robust outcome for this study.

The regression discontinuity design estimates only the effect of an intervention at the point of the discontinuity (here, $34+0$ weeks). Thus, our conclusions are most generalizable to gestational ages close to $34+0$ weeks, and we caution that our conclusions may not apply to markedly different gestational ages (e.g., term gestation). Although the rate ratios from our models suggest that antenatal corticosteroids do not increase the risk of ADHD, the upper limits of our $95 \% \mathrm{Cls}$ preclude conclusively ruling out a moderate degree of increased risk. However, the upper limits of our Cls are well below the magnitude of risks found in previous studies that generated current safety concerns, such as the 2.1-fold increase in risk of being in the lowest quartile of academic ability, as observed in the ASTECS trial, ${ }^{14}$ or the (nonsignificant) 5.7 -fold increase in risk of cerebral palsy observed in a trial of repeat courses of antenatal corticosteroids. $^{33}$

Finally, the regression discontinuity design depends on an assumption that exposure to antenatal corticosteroids was high among infants just before the clinical cut-off of $34+0$ weeks, and low among infants just after (an assumption analogous to the need for high treatment adherence in a randomized trial). Our ability to document corticosteroid administration was limited by a known shortcoming in the Perinatal Registry variable for antenatal corticosteroids, which instructs coders not to indicate administration in cases where documentation on timing of administration is incomplete. We estimate that about $75 \%$ of pregnancies admitted for delivery before 34 weeks' gestation were exposed to corticosteroids (based on an unpublished chart audit of BC infants before 34 weeks' gestation, ${ }^{34}$ and previously published national data of neonatal intensive care units from 2003-2013), ${ }^{35}$ but cannot confirm this empirically. We also had no information on the timing or number of doses administered; it is likely that some of the pregnancies that received antenatal corticosteroids had incomplete doses or suboptimally timed administration.

\section{Conclusion}

We did not identify an association between higher probability of antenatal corticosteroid exposure and prescriptions for ADHD medications in childhood. Our findings may be useful in supporting discussions with families about the harms and benefits of antenatal corticosteroid administration, particularly at late preterm gestations, where the need for safety data is most pressing.

\section{References}

1. National Institutes of Health. Effect of corticosteroids for fetal maturation on perinatal outcomes. NIH Consens Statement 1994;12:1-24.

2. Antenatal corticosteroids to reduce neonatal morbidity and mortality. London (UK): Royal College of Obstetricians and Gynaecologists; 2010.

3. Gyamfi-Bannerman C, Thom EA, Blackwell SC, et al. Antenatal betamethasone for women at risk for late preterm delivery. N Engl J Med 2016;374:1311-20.

4. Crowther CA, Harding JE. Antenatal glucocorticoids for late preterm birth? N Engl J Med 2016;374:1376-7.

5. Jobe $\mathrm{AH}$, Goldenberg RL. Antenatal corticosteroids: an assessment of anticipated benefits and potential risks. Am J Obstet Gynecol 2018;219:62-74.

6. Yee W, Amin H, Wood S. Elective cesarean delivery, neonatal intensive care unit admission, and neonatal respiratory distress. Obstet Gynecol 2008;111:823-8. 
7. De Luca R, Boulvain M, Irion O, et al. Incidence of early neonatal mortality and morbidity after late-preterm and term cesarean delivery. Pediatrics 2009; 123:e1064-71.

8. Nowik CM, Davies GA, Smith GN. We should proceed with caution when it comes to antenatal corticosteroids after 34 weeks. J Obstet Gynaecol Can 2017; 39:49-51.

9. Smith GC, Rowitch D, Mol BW. The role of prenatal steroids at 34-36 weeks of gestation. Arch Dis Child Fetal Neonatal Ed 2017;102:F284-5.

10. DeMauro SB. Antenatal corticosteroids: Too much of a good thing? JAMA 2020;323:1910-2

11. Effects of antenatal dexamethasone administration in the infant: long-term follow-up. J Pediatr 1984;104:259-67.

12. Dalziel SR, Lim VK, Lambert A, et al. Antenatal exposure to betamethasone: psychological functioning and health related quality of life 31 years after inclusion in randomised controlled trial. BMJ 2005;331:665.

13. Dessens AB, Haas HS, Koppe JG. Twenty-year follow-up of antenatal corticosteroid treatment. Pediatrics 2000;105:E77.

14. Stutchfield PR, Whitaker R, Gliddon AE, et al. Behavioural, educational and respiratory outcomes of antenatal betamethasone for term caesarean section (ASTECS trial). Arch Dis Child Fetal Neonatal Ed 2013;98:F195-200.

15. Sotiriadis A, Tsiami A, Papatheodorou S, et al. Neurodevelopmental outcome after a single course of antenatal steroids in children born preterm: a systematic review and meta-analysis. Obstet Gynecol 2015;125:1385-96.

16. Raikkonen K, Gissler M, Kajantie E. Associations between maternal antenatal corticosteroid treatment and mental and behavioral disorders in children. JAMA 2020;323:1924-33.

17. Owen D, Matthews SG. Repeated maternal glucorticoid treatment affects activity and hippocampal NMDA receptor expression in juvenile guinea pigs. $J$ Physiol 2007;578:249-57.

18. Crowther CA, Doyle LW, Haslam RR, et al. Outcomes at 2 years of age after repeat doses of antenatal corticosteroids. N Engl J Med 2007;357:1179-89.

19. Moscoe E, Bor J, Barnighausen T. Regression discontinuity designs are underutilized in medicine, epidemiology, and public health: a review of current and best practice. J Clin Epidemiol 2015;68:122-33.

20. Guduguntla V, McWilliams JM. Exploiting clinical decision-making thresholds to recover causal effects from observational data: randomization without trials. JAMA Intern Med 2021;181:774-5.

21. Crane J, Armson A, Brunner M, et al.; Society of Obstetricians and Gynaecologists of Canada Expert Opinion. Antenatal corticosteroid therapy for fetal lung maturation. J Obstet Gynaecol Can 2003;25:45-52.
22. Hutcheon JA, Harper S, Liauw J, et al. Antenatal corticosteroid administration and early school age child development: a regression discontinuity study in British Columbia, Canada. PLoS Med 2020;17:e1003435.

23. British Columbia Ministry of Health. PharmaNet V2. Vancouver: Population Data BC; 2018. Available: https://www.popdata.bc.ca/data/health/pharmacare (accessed 2018 May 1).

24. The British Columbia Perinatal Data Registry. Vancouver: Perinatal Services BC; 2018. Available: https://www.popdata.bc.ca/data/health/perinatal (accessed May 1).

25. BC Vital Statistics Agency. Vital statistics deaths V2. Vancouver: Population Data BC; 2018. Available: https://www.popdata.bc.ca/data/demographic/vs_ deaths (accessed 2018 May 1).

26. British Columbia Ministry of Health. 2018 Consolidation file (MSP registration and premium billings) V2. Vancouver: Population Data BC; 2018. Available: https://www.popdata.bc.ca/data/demographic/consolidation_file (accessed 2018 May 1).

27. Delaney M, Roggensack A; Clinical Practice Obstetrics Committee. guidelines for the management of pregnancy at $41+0$ to $42+0$ weeks. J Obstet Gynaecol Can 2008;30:800-10.

28. Frosst G, Hutcheon J, Joseph KS, et al. Validating the British Columbia Perinatal Data Registry: a chart re-abstraction study. BMC Pregnancy Childbirth 2015;15:123.

29. Feldman ME, Charach A, Bélanger SA. Position statement: ADHD in children and youth: part 2 - treatment. Paediatr Child Health 2018;23:462-72.

30. O'Keeffe AG, Geneletti S, Baio G, et al. Regression discontinuity designs: an approach to the evaluation of treatment efficacy in primary care using observational data. BMJ 2014;349:g5293.

31. Smith LM, Levesque LE, Kaufman JS, et al. Strategies for evaluating the assumptions of the regression discontinuity design: a case study using a human papillomavirus vaccination programme. Int J Epidemiol 2017;46:939-49.

32. Hauck TS, Lau C, Wing LLF, et al. ADHD Treatment in primary care: demographic factors, medication trends, and treatment predictors. Can J Psychiatry 2017;62:393-402.

33. Wapner RJ, Sorokin Y, Mele L, et al. Long-term outcomes after repeat doses of antenatal corticosteroids. N Engl J Med 2007;357:1190-8.

34. Kazem M, Hutcheon JA, Joseph KS. A population-based study of antenatal corticosteroid prophylaxis for preterm birth. J Obstet Gynaecol Can 2012;34:842-8.

35. Melamed N, Murphy K, Barrett J, et al. Benefit of antenatal corticosteroids by year of birth among preterm infants in Canada during 2003-2017: a populationbased cohort study. BJOG 2021;128:521-31.
Competing interests: Amanda Skoll is chair of the examination committee with the Royal College of Physicians and Surgeons of Canada. Myriam Srour reports grants from the Canadian Institutes of Health Research and the Montréal Children's Foundation, outside the submitted work. No other competing interests were declared.

This article has been peer reviewed.

Affiliations: Department of Obstetrics \& Gynaecology (Hutcheon, Liauw, Skoll), University of British Columbia, Vancouver, BC; Department of Epidemiology, Biostatistics, and Occupational Health (Strumpf, Socha, Harper), Economics (Strumpf), Pediatrics (Srour), and Neurology and Neurosurgery (Srour), McGill University, Montréal, Que.; Department of Pediatrics (Ting), University of British Columbia, Vancouver, BC

Contributors: Jennifer Hutcheon, Erin Strumpf and Sam Harper conceived of the study. Jennifer Hutcheon, Erin Strumpf, Jessica Liauw, Amanda Skoll, Myriam Srour and Sam Harper designed the study. Jennifer Hutcheon acquired and analyzed data. All authors interpreted findings. Jennifer Hutcheon wrote the first draft of the manuscript. All of the authors revised it critically for important intellectual content, gave final approval of the version to be published and agreed to be accountable for all aspects of the work.

Content licence: This is an Open Access article distributed in accordance with the terms of the Creative Commons Attribution (CC BY-NC-ND 4.0) licence, which permits use, distribution and reproduction in any medium, provided that the original publication is properly cited, the use is noncommercial (i.e., research or educational use), and no modifications or adaptations are made. See: https://creativecommons.org/ licenses/by-nc-nd/4.0/

Funding: This research was funded by the Canadian Institutes of Health Research. Jennifer Hutcheon holds a Canada Research Chair in Perinatal Population Health. Myriam Srour holds a Chercheur-Boursier award from the Fonds de recherche du Québec - Santé. Erin Strumpf holds a William Dawson Scholar award at McGill University. Peter Socha is supported by a graduate training award from the Fonds de recherche du Québec - Santé. Funders had no role in the design, conduct or reporting of this study.

Data sharing: The data used in this study cannot be shared by the research team, but can be accessed by submitting a data request to Population Data BC (https://www.popdata.bc.ca/data_access).

Acknowledgements: The authors thank Tim Choi from Population Data BC for his invaluable assistance in data access. All inferences, opinions and conclusions drawn in this study are those of the authors, and do not reflect the opinions or policies of the data stewards.

\section{Accepted: Dec. 16, 2021}

Correspondence to: Jennifer Hutcheon, jhutcheon@bcchr.ca 